Session 1658

\title{
Knowledge Management for Enterprise Applications Integration
}

\author{
Melissa C. Lin \\ Information Technology Consultant
}

\begin{abstract}
During the past economic boom years, major companies invested heavily in enterprise systems, communication bandwidth, Internet technologies, and the Y2K redemption. Therefore, creating a strong foundation of enterprise information integration becomes one of the major factors in reducing cost and enhancing services. This paper discusses knowledge management techniques for enterprise information integration, and includes data integration, business-process integration, and method-procedure integration that link multiple applications and database to share information and business process. The paper describes significant and extensive coordination and integration of enterprise applications, including technical and administrative that directly effect the organization and the applications managed.
\end{abstract}

\section{Introduction}

With the current fast paced advancement of Information Technologies, many companies have worked on information technology integration and built user-center enterprise information systems for their employees and customers, in order to reduce the cost and enhance the services. The integration of enterprise applications basically involves people, processes, applications, and information. A modern enterprise information application system assists in gathering certain data routinely (such as economic or health statistics) in order to develop policies and to analyze history data for marketing forecasting, sales trend, etc. It also includes dealing with E-mails, inventory lists, visitor counts, equipment maintenance reports, spending records, etc. The knowledge of how to link multiple applications and database becomes significant, when managing Enterprise Information Technology. A successful knowledge management of enterprise integration helps make information simple and clear to follow and adopt, legitimizes the knowledge with the team workforce, and allows the business intelligence encompass a wide range of products. Getting people to optimize a knowledge management with information technologies is a continuous effort. As a greater number of people adopt it, they will look for more opportunities to share and collaborate in other ways. 
Typical enterprise applications, including ERP (Enterprise Resource Planning), CRM (Customer Relationship Management), SCM (Supply Chain Management), and ERM (Employee Relationship Management), require various types of integration of business process, applications, and information. The four distinct forms of integration and examples reported in [1] include

a) Portals integration - the shallowest form of integration, which brings potentially dissimilar applications together in a single Web-based portal entry point.

b) Business-process integration - a form of integration that coordinates business processes across applications and around enterprise boundaries. One good example is a supply chain management system. It is observed that Web services [3] using XML-based protocols such SOAP (Simple Object Access Protocol) [4], WSDL (Web Service Definition Language) [5], and UDDI (Universal Description, Discovery, and Integration) [6] and their derivatives are becoming important here.

c) Application integration - a form of integration that focuses on data transformation and message queuing to allow similar or complementary applications to communicate with each other. It is increasingly used in the XML (Extensible Markup Language) [2] domain.

d) Information integration - a form of integration that brings together complementary data that is either physically or logically related. In addition for allowing applications to be written to, it also makes it possible for the application to use all of the relevant data in the enterprise. A customer relationship management (CRM) application is an example of this.

This paper discusses how to turn on these "know how" information technologies into productivity engines for Enterprise Applications Integration. It includes creating, managing, integrating, web-enabling, and delivering unstructured digital content across the enterprise and beyond - to employees, customers and trading partners - that creates real business value. Therefore, the paper discusses enterprise information systems and Web services, the Integration of Enterprise Applications, as well as how to manage technical and non-technical conflicts in the process of enterprise applications integration and how to motivate people with knowledge management.

\section{Enterprise Information Systems and Web Services}

The Web is evolving from a pure document repository into a provider of information and computing services. Traditional Web-based systems and services are built on existing Web servers with an integration of technologies using CGI methods and/or other server-side programming techniques. Figure 1 shows the files and directories of a typical Apache Web server and database server for supporting typical Web-enabled tasks including HTML (HyperText Markup language) page posting, dynamic HTML pages (JavaScript, Java Applet, ActiveX, etc) generating and parsing HTML and XML (eXtensible Markup Language) documents. 


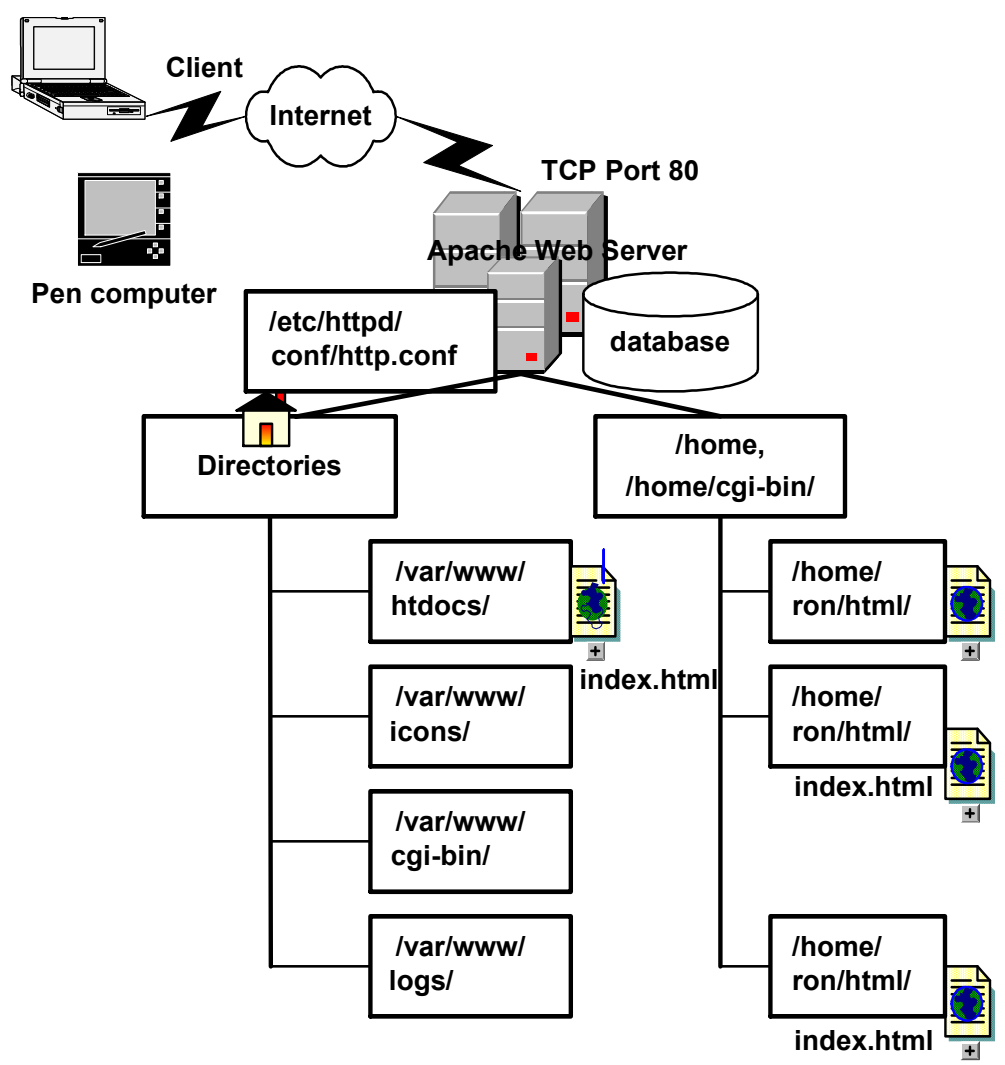

Figure1 - A Linux-Based Apache Web Server

As Web technologies advance, the World Wide Web (WWW) is used to deliver IT infrastructure, application development, application hosting and service, application maintenance, and end-user software. Therefore, the standardized programmatic interfaces for remote invocation of software objects among heterogeneous computer systems over the Internet are desired. Most Web-enabled database applications are generally accomplished through CGI (common gateway interface) and server-side programming using such programming tools as Perl, C/C++, VB, VB.NET, ASP.NET, Java Servlet, etc. Figure 2 shows a Java-based architecture for Java-based Web-enabled database application.

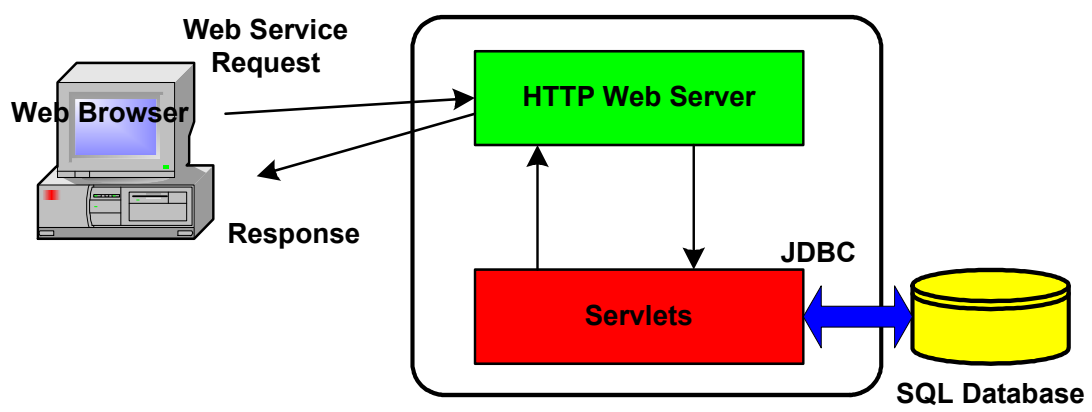

Java Web Server Using Servlets and JDBC

Figure 2 - A Typical Java-based Web-Enabled Database Applications

Proceedings of the 2004 American Society for Engineering Education Annual Conference \& Exposition Copyright@2004, American Society for Engineering Education 
A Web Service is a network accessible interface to application functionality, built using standard Internet technologies. Web services are programmatic interfaces for applicationto-application communication using a combination of protocols like HTTP, HTML, XML, SOAP, WSDL, UDDI, etc. Web services are accompanied by information explaining what they do and how other applications can access and use them. The World Wide Web Consortium (www.w3c.org) is responsible for developing interoperable technologies including specifications, guidelines, software, and tools for Web-based applications.

Web services can be implemented, without requiring radical rework, to provide enhanced business opportunities and ultimately lower cost. Some known advantages of Web Services may include

a) An enabling architecture for wide-area distributed computing among heterogeneous computer systems over the Internet.

b) Distributed information technology knowledge and data based on the XML standard

c) Using XML-based languages for developing Web service objects, (collection of data and methods) with predefined interfaces for accessing data and methods

d) Supporting remote method invocation.

The architecture of Web Services includes four main components - Web service providers, Service description, Web service requesters, and Web service registry servers, as shown in the Figure 3.

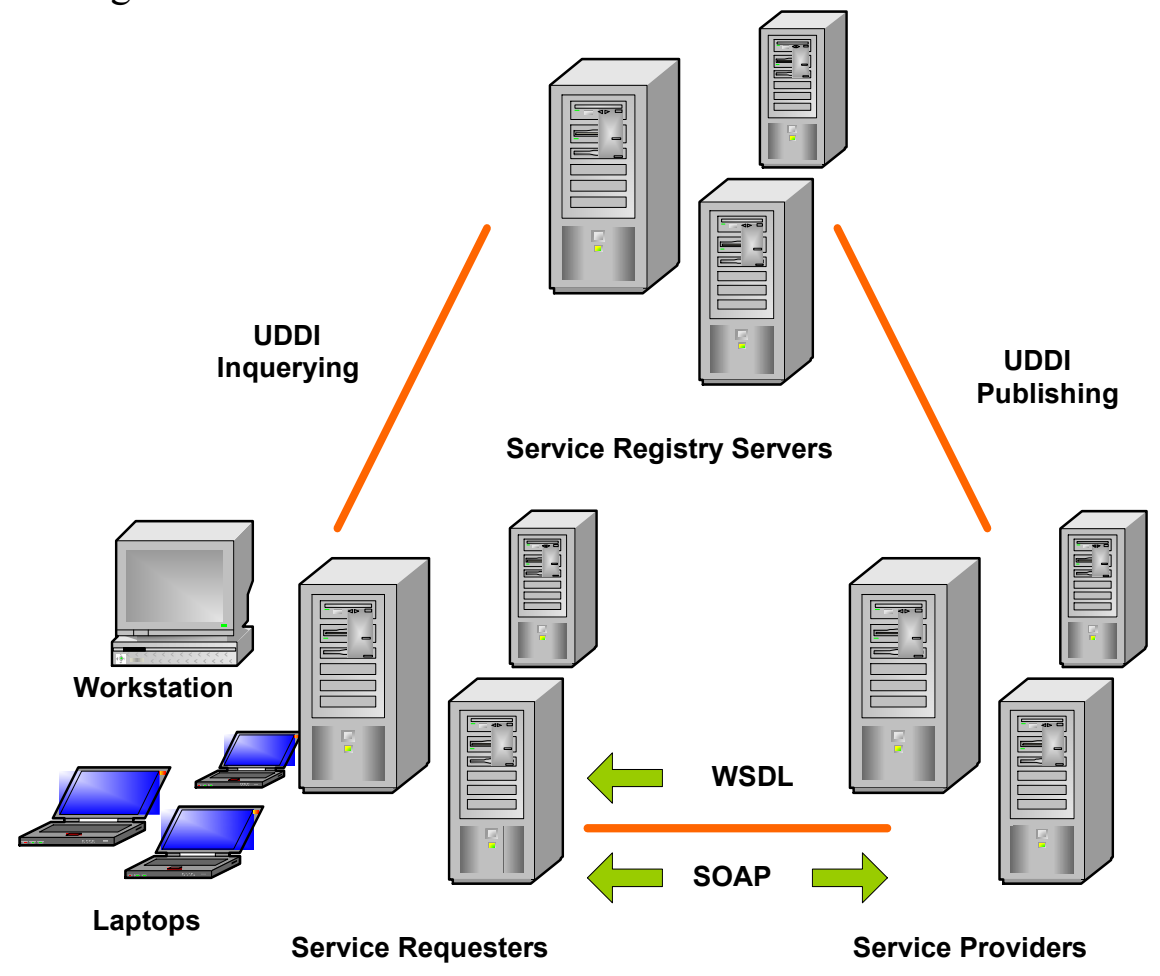

Figure 3 - Main Components of Web Service Architecture 
Web service providers produce, test, and publish SOAP-based (Simple Object Access Protocol) Web services on the Internet. Service description uses WSDL (Web service Description Language) definitions to describe Web services. Web service requesters use XML-based SOAP clients based on the WSDL and searching for published services in UDDI (Universal Description, Discovery, and Integration) directory. Web service registry servers (in a centralized UDDI registry) are located within centralized UDDI registries.

Web Service and related technologies can be deployed to provide access to business functions such as business logic execution, business intelligence, on-line analysis, analytic software, front-end and back-end hardware interface, business automation, instant messaging, etc., over the Web. From the technology point of view, Web Service can also address the challenges of B2B process and relieve the difficulty in implementing technologically complex B2B systems that may require various data interchange operations and Remote Procedure Calls (RPC).

Data interchange presents problems because mapping data formats across vendors and platforms is a daunting task. A change in data format at one location can wreak chaos throughout the system. RPC poses a different challenge: regardless of the technologies employed or the business functions mapped, each process a company automates requires an exercise in specifying and documenting a new RPC. Each new customer requires a new connection, a new construct, and a new complex interface. XML and Web Services-based strategies and IT architectures address these issues occurred in both data interchange and RPC. XML has found its way into a multitude of products and every major platform for describing data. Web Service offers a practical solution to the discovery of XML-RPC interfaces [7].

Another advantage of Web Services is that it provides a standard of linking all applications via SOAP messages to create reusable interfaces for Enterprise Applications. A SOAP message consists of an envelope containing an optional header and a required body. It is designed to sit between a service consumer and a service provider, and then add value or functionality to the transaction between the two. IBM has also supplied an extension to Apache SOAP [8] called the Web Services Toolkit that provides comprehensive Web Service Definition Language (WSDL) support on top of Apache SOAP. Many Enterprise Applications Integration vendors are enhancing their products to support Web services standards, such as SOAP, WSDL, and UUDI. Although Web services technology can enable Enterprise Application Integration, it does not constitute an enter Enterprise Application Integration solution. Not all integration issues can be solved using Web services. Web service might not provide the levels of security, reliability, performance, and uptime required by some organizations. 


\section{Enterprise Applications Integration (EAI)}

Enterprise application integration has been the driving force behind application and information system development for the last few years. The EAI is expected to provide seamless integration of Business Systems for sharing and exchanging data between dissimilar systems, and conducting business electronically. Because many services are now provided through the Web, it is essential that business enterprises have an efficient Webbased EAI solution which allows other Web applications to access and use the legacy application's data, and supports every aspect of data and application interacts between an organization and its customers - including marketing, sales, service and support, etc. Figure 4 shows a small-scale Java-based EAI architecture with such key components JIN (Java Native Interface) for linking to legacy systems, JDBC (Java database connectivity) for communicating to database systems, and RMI (Remote Method Invocation) for calling procedures. This system can use additional Java support for Enterprise Application Integration (EAI) and for linking Enterprise Information Systems (EISs) with Web services.

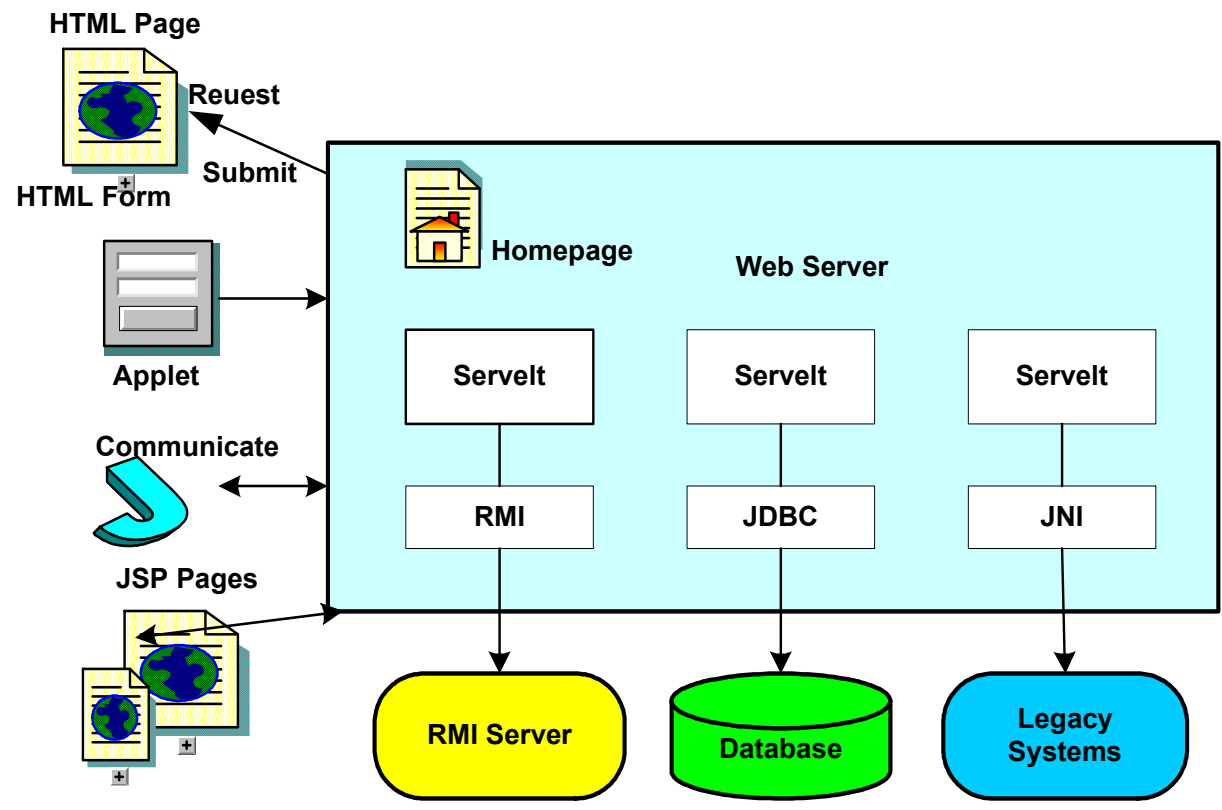

Figure 4 - A Small Scale EAI Infrastructure Build on Java Technology

One of the important issues in EAI is enterprise data sharing and data exchanging, which requires Web servers to communicate in between Web browsers and Database. Figure 5 shows an example of EAI infrastructure, which includes appropriate adapter modules for data integration, business-process integration, and method-procedures integration. Data integration involves linking databases to applications and/or other databases. Businessprocess integration and method-procedures integration involves connecting applications so that they can communicate and access each other's functionality. To facilitate business processes and ensure maximum security, many companies employ Web services to supply users with personalized information, and link with disparate applications and data sources. 


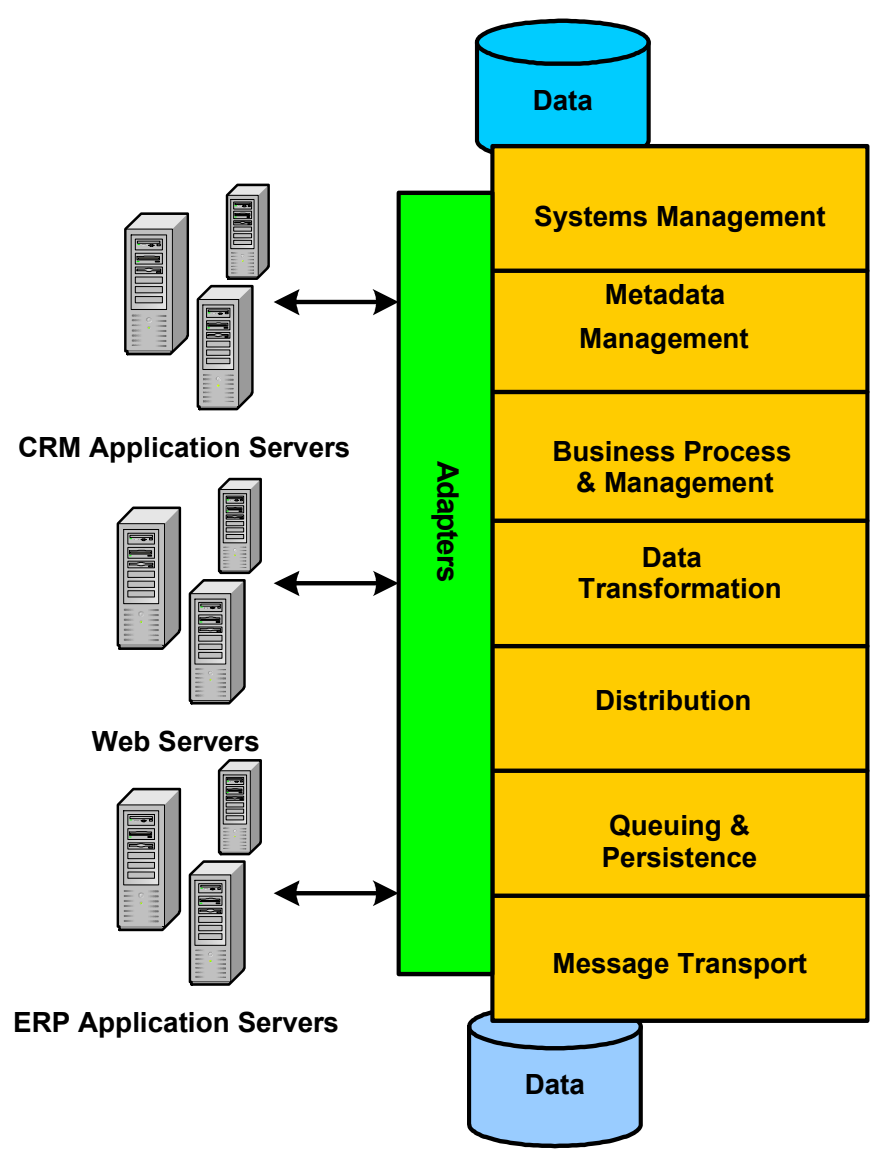

Figure 5 - An Example of Enterprise Applications Integration

\section{Managing Conflict in Enterprise Application Integration}

Enterprise Application Integration involves many people and software tools, each focusing on some aspect of the overall process. Although many software tools and automated methods are available, the development of integration ultimately depends on the interpersonal interactions of the people dealing with people and tools. Technical and nontechnical conflicts occasionally arise from involved staff not sharing the same goals or understanding. Most conflicts arise in organizations for a variety of reasons, including scarce resources, interdependent work, differentiated work, competitive reward systems, perceptions of inequity, and asymmetrical distribution of power. These factors often have the potential to interfere with work performance and integration quality. Managing conflict includes identifying the collective skills of employees, identifying the problems of the environment, understanding how it affects work processes and outcomes, and resolving it with appropriate manners.

One of the most common issues of conflict develops through competition between colleagues. Therefore, "Finding a Right person for an appropriate need" and "Finding a 
Right enterprise tool" are keys of managing conflict and affecting the most return value in performance.

Because conflicts have a significant impact on the managing and integration of enterprise applications, knowing how to reduce conflicts between people and tools is essential. A few tips of managing conflicts are shown in [11]:

- Identify the business requirements and analyzing the current applications

- Select appropriate software tools to develop technical solutions to meet the needs

- Emphasize status reports and support the integration of enterprise applications in order to save time.

- Managing sustainable technology, applications, process, people, and organization is necessary, allowing one to control costs and enhance service.

- Good communication and interpersonal skills are required to resolve or improve the overall development process.

\section{Motivation of Knowledge Management}

Knowledge management is an evolving broad umbrella of topics and viewpoints, which makes a comprehensive look at the subject difficult. The approach requires focusing on high quality links from executive offices and web sites, which encompass most of the major trends. The knowledge management for enterprise applications integration reduces conflicts between people and applications, allows sharing of "know how" information technology into productivity engines, utilizes automatic data processing methods to improve operations, and sets and adjusts priorities of workloads for the Enterprise Applications. As shown in Figure 6, we can use knowledge management to drive relationships between colleagues and customers, encourage feedback, foster a frank environment where our employees are not afraid to speak their minds, and to promote motivation of working abilities. We need to create a culture that makes employees comfortable with adding suggestions and presenting subject-related ideas. From their ideas and questions, we are able to understand and manage their learning schedule and provide the necessary training. The willingness and motivation of the Information Technology professionals is a key factor in learning and sharing knowledge. Here are the communication tips of knowledge management [12]:

- Creating a culture where employees are not punished for asking what leaders do not want to hear

- Appointing meeting and group leaders to whom the employees perceive as credible and trustworthy

- Getting employees to communicate from the bottom up

- Being able to answer questions

- Asking what do I expect to get out of this communication vehicle?

- Scheduling brown-bag lunches to work best with people of similar levels

- Establishing an incentive program when you give me X, I will give you Y 


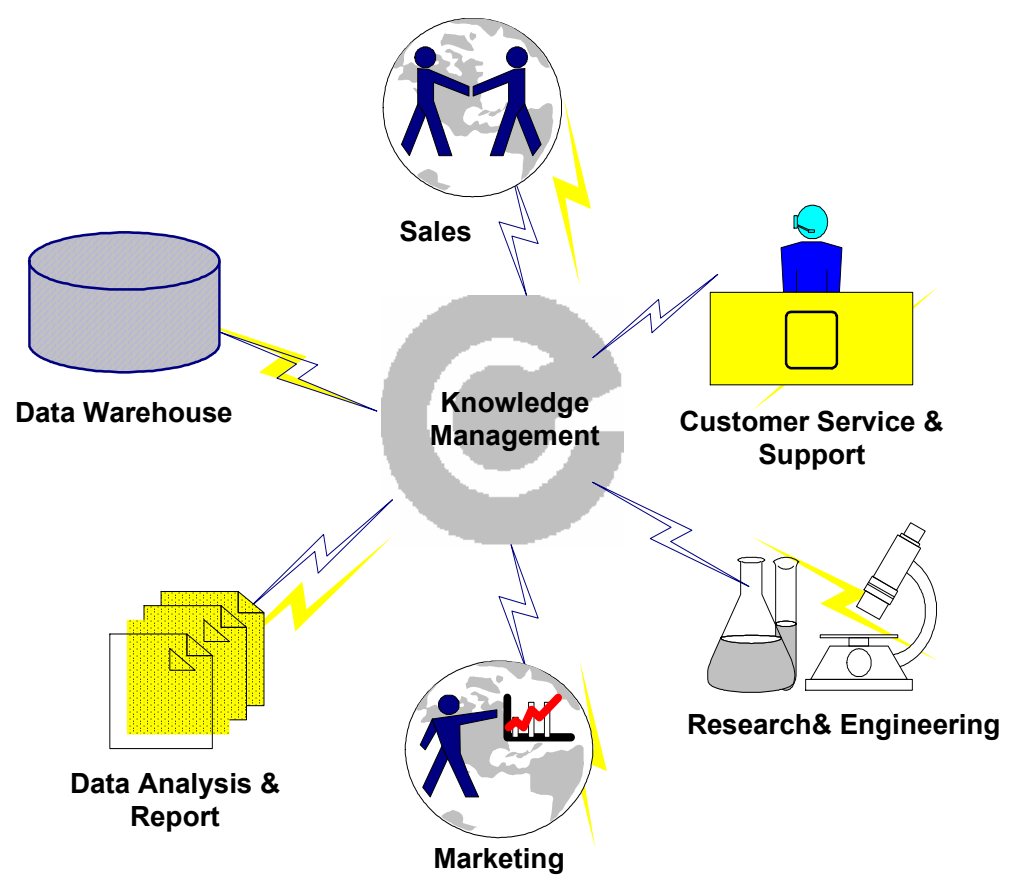

Example 6 - Knowledge Management and EAI

Once we have defined the strategy and needs of knowledge management, we are ready to identify the employees who are most likely to fit our needs. Finding a Right person to deliver technical knowledge between colleagues and customers is very important. As more employees understand exactly what is offered by the enterprise application, better results will be achieved. Here are the motivation tips of knowledge management [12]:

- Knowing how to work well in teams

- Showing enthusiasm for the job

- Understanding the business mission behind Information Technology initiatives

- Having a strong work ethic

- Thinking creatively to solve problems

- Making a good impression with the entire IT team

- Making a good impression with the IT organization's customers

- Being highly intelligent and having excellent problems solving abilities

- Having relevant experiences and education

The integration of enterprise applications used varies significantly from organization to organization. Knowledge management has its appropriate values for each application in order to remain competitive and avoid the hazards of outsourcing. To this end, the Information Technology team uses knowledge management to select a suitable business intelligence solution, manage technical and non-technical conflict, and share knowledge that is efficient, improves decision-making, and is easy to maintain, yet flexible and powerful. 


\section{Conclusion}

Knowledge management will assist employees in performing various tasks, from retrieving corporate policies or human-resource information to scheduling work related trips and accessing applications. Knowledge Management for Enterprise application Integration is one of solutions that can resolve the managing conflict in networking, administration and project management, operating systems and hardware platforms, multiple software products, and software development for multiple platforms. Once we turn the "Know How" information technologies into productivity engines for Enterprise Applications, it will enhance customer relationship and services as well as reduce the cost of expenses across multiple industries. The key to knowledge management is encouraging people to become motivated and learn, while working as a team.

\section{References}

1. A.D. Jhingran, N. Mattos, and H. Pirahesh, "Information Integration: A Research Agenda,” IBM System Journal, Vol 41, No. 4, 2002, pp. 555-562, http://www.research.ibm.com/journal/sj/414/jhingran.pdf.

2. Extensible Markup Language (XM), http://www.w3.org/XML/.

3. Web Service Description Working Group, http://www.w3.org/2002/ws/desc/

4. SOAP Version 1.2, http://www.w3.org/2000/xp/Group/.

5. Web Service Description Language, http://www.w3.org/TR/wsdl

6. UDDI of Web Services Operation Council, www.uddi.org.

7. XML-RPC Specification, http://www.xmlrpc.com/spec

8. RPC \& e-Commerce, www.w3.org/2000/03/xp65435/RPC AND eCommerce.ppt

9. Deitel developer series, "Web Services A Technical Introduction," Prentice Hall, 2003.

10. Enterprise Application Integration Consortium, http:/www.eaiindustry.org/

11. "Managing Conflict in Software Testing," Communication of the ACM, January 2004.

12. "Special Report - Turn on the Know How," Federal Computer Week, October 20, 2003.

13. "Premier 100 IT Leaders 2004," Computer World, Jan 5, 2004.

14. Paul I-Hai Lin, "XML and Enterprise Applications Integration," Indiana-Purdue University at Fort Wayne, 2003, http://www.etcs.ipfw.edu/ lin

15. M.A. Roth, et. al, "Information Integration: A New Generation of Information Technology," IBM System Journal, Vol 41, No. 4, 2002, pp. 563-577, http://www.research.ibm.com/journal/sj/414/cody.pdf.

16. W.F. Cody, et. al, "The Integration of Business Intelligence and Knowledge Management,” IBM System Journal, Vol 41, No. 4, 2002, pp. 697-713, http://www.research.ibm.com/journal/sj/414/cody.pdf

17. Apache Web Services Project, http://ws.apache.org

18. Java Servlet Technology, http://java.sun.com/products/servlet/archive.html

19. Java Technology - The Source for Developer, http://developers.sun.com/

20. JDBC Basics, http://java.sun.com/docs/books/tutorial/jdbc/basics/

21. Java Remote Method Invocation, http://java.sun.com/products/jdk/rmi/

22. Java Native Interface, http://java.sun.com/j2se/1.3/docs/guide/jni/

23. ebXML- Electronics Business using XML, www.ebXML.org

Proceedings of the 2004 American Society for Engineering Education Annual Conference \& Exposition Copyright 02004 , American Society for Engineering Education 


\section{Biographical}

Melissa Lin is an IT Consultant - eLearning \& Distance instructor at HenEm in 2004. Previously, she was an Information Technology Specialist at Farm Service Agency, USDA in 2003, an IT Consultant - CRM at Exactech in 2002, a Database Administrator - Data Warehouse at University of Florida in 2000 - 2002, a Visiting Professor in the Computer Science Department of Indiana University - Purdue University, Fort Wayne in 1999 - 2000. Before that, she worked as systems analyst at GTE Data Service, Fort Wayne, Indiana, for 11 years, responsible for E911 and CBSS billing supports. Lin's current interests are leadership communication, knowledge management in enterprise applications including information technology and customer relationship. 